1 Fundação Oswaldo Cruz (Fiocruz), Escola Nacional de Saúde Pública Sérgio Arouca (Ensp) - Rio de Janeiro (RJ), Brasil. giovanel@ensp.fiocruz.br

2 Universidade Federal Fluminense (UFF), Instituto de Saúde Coletiva, Departamento de Planejamento em Saúde Niterói (RJ), Brasil. patty.fidelis@hotmail.com

3 Pontificia Universidad Javeriana - Bogotá, Colombia.

rrvega.romero@gmail.com

${ }^{4}$ Centro Universitário Uniabeu - Belford Roxo (RJ), Brasil.

suelen.c.oliveira@gmail.com

5 Universidad Mayor de San Andrés - La Paz,

Bolivia.

herland.tejerina@gmail.com

*Participaran igualmente como coautores: Gilberto Ríos, Naydú Acosta

Ramírez y Hedwig Goede.

\section{Panorama de la Atención Primaria de Salud en Suramérica: concepciones, componentes y desafíos}

\author{
Overview of Primary Health Care in South America: conceptions, \\ components and challenges
}

\author{
Ligia Giovanella', Patty Fidelis de Almeida², Román Vega Romero ${ }^{\mathbf{3}}$, Suelen Oliveira4, Herland \\ Tejerina Silva ${ }^{5^{\star}}$
}

RESUMEM El artículo presenta un panorama de la Atención Primaria de Salud en Suramérica a partir de mapeos realizados en los 12 países según una matriz analítica organizada en las dimensiones: conducción, financiamiento, características de la prestación y organización, coordinación de cuidados e integración a la red de servicios, fuerza de trabajo, participación social, acción intersectorial e interculturalidad. Se observa diversidad en la implementación y de abordajes de APS, condicionada por directrices políticas, modalidades de protección social y segmentación de los sistemas de salud. Se destacan iniciativas innovadoras de atención primaria integral y se identifican los principales desafíos.

PALABRAS CLAVE Atención Primaria de Salud; Atención integral de salud; Sistemas de salud.

\begin{abstract}
The article presents an overview of Primary Health Care in South America. Developed from mappings in the 12 countries, it follows an analytical matrix organised into the following parameters: stewardship, funding, characteristics of provision and organisation, care coordination and integration with the service network, labour force, social participation, inter-sector collaboration and intercultural approaches. Diversity was observed in PHC implementation and approaches, which are conditioned by policy guidelines, social protection modalities and health system segmentation. Innovative initiatives in comprehensive $P H C$ are highlighted and the main challenges are identified.
\end{abstract}

KEYWORDS Primary Health Care; Comprehensive health Care; Health systems. 


\section{Introducción}

En América del Sur, desde la Declaración de la Conferencia Mundial de Alma-Ata sobre Atención Primaria de Salud (APS) en 1978, persisten tensiones entre distintas formas de concebir y abordar la implementación de la APS en los países a lo largo del tiempo. La Declaración de Alma-Ata difundió una concepción de atención primaria de la salud integral, que la interpreta como parte de un sistema integrado de cuidados de la salud y del desarrollo económico-social de una sociedad, que involucra la cooperación con otros sectores para enfrentar los determinantes sociales de la salud y la promoción de la participación social.

La concreción de estos principios varía en cada país y los abordajes de APS en Suramérica presentaron distintos énfasis en las últimas décadas conforme a los diversos contextos políticos y modalidades de sistemas de protección social y de sistemas de salud prevalentes (CONILL; FAUSTO; GIOVANELLA, 2010; ROVERE, 2012; TEJERINA SILVA ET AL., 2009).

En los años 1980 y 1990, en diversos países de América del Sur, en los contextos autoritarios y de ajustes macroeconómicos estructurales, se adoptaron modelos de APS selectivos y focalizados promovidos por agencias financieras multilaterales que propugnaban la reducción del rol del sector público y la implementación de paquetes mínimos de servicios de salud, dirigidos a grupos poblacionales marginados (LABONTÉ ET AL., 2009). Se reforzaron programas verticales orientados a grupos poblacionales o problemas de salud específicos con la creación de seguros focalizados, lo que profundizó la segmentación en los sistemas de salud de los países de la región y las inequidades sociales.

En la primera década del siglo XXI, con los cambios políticos en procesos de redemocratización y asunción de gobiernos más comprometidos con la justicia social, se observa un proceso de revitalización de la APS en su abordaje integral de acuerdo con la concepción de Alma-Ata. Se busca la construcción de sistemas de salud orientados por una APS integral como coordinadora de los cuidados en una red integrada de servicios $\mathrm{y}$ articuladora de acciones comunitarias e intersectoriales que incidan sobre los determinantes sociales para promover la salud y garantizar el derecho universal al acceso a los servicios de salud.

Al mismo tiempo, agencias internacionales de salud como la Organización Panamericana de Salud (OPS, 2005) y la Organización Mundial de la Salud (OMS, 2008) instaron a los países miembros a renovar la APS para lograr la cobertura universal.

Las características y magnitud de estos procesos de renovación de la APS en la región aún son poco conocidos, en parte por su historia reciente, en parte por el esfuerzo de investigación que implica abarcar procesos en diversos países. El objetivo del presente artículo es ofrecer un panorama de la APS en los 12 países de América del Sur, analizar los abordajes, sus condicionantes y los desafíos para implementar una atención primaria integral de salud en la región. Se busca identificar las concepciones que orientan los modelos asistenciales en implementación en los países de Suramérica y explorar la hipótesis de que las modalidades de protección social en salud y la segmentación de los sistemas de salud condicionan la concepción de APS implementada y obstaculizan la implementación de nuevos modelos de APS integral.

\section{El mapeo de la APS en Suramérica}

Este artículo presenta los principales resultados de un mapeo sobre la APS realizado en los 12 países de América de Sur por el Instituto Suramericano de Gobierno en Salud (Isags), organismo internacional del Consejo de Salud Suramericano, de la Unión de Naciones Suramericanas (Unasur). 
Se realizaron estudios de caso en cada uno de los 12 países de América del Sur con base en una metodología similar, incluyendo múltiples estrategias de recolección de datos e informaciones: revisión de literatura, análisis de documentos, visitas a ministerios de salud y servicios de APS. Con el apoyo de los representantes nacionales del Grupo de Trabajo en Sistemas Universales de Unasur, fueron organizadas y realizadas visitas a los países con entrevistas en los ministerios de salud, relevamiento de datos y documentos y observación in loco de experiencias y servicios de APS seleccionados por los ministerios de salud en el período de noviembre de 2013 a abril de 2014.

La recolección de información para los mapeos de APS en cada caso fue realizada en base a una matriz común. La construcción de la matriz y la definición de sus dimensiones se basan en la concepción de atención primaria integral de la Declaración de AlmaAta y se buscó operacionalizar sus principios fundamentales.

La matriz está organizada en dimensiones: conducción de la APS, financiamiento de la APS, características de la prestación y de la organización de la APS, coordinación de los cuidados e integración de la APS en la red de servicios, fuerza de trabajo en la APS, participación social, actividades intersectoriales e interculturalidad. Para cada dimensión se definieron componentes, sintetizados en el cuadro 1. Las desigualdades regionales y sociales en el acceso a la APS fueron tomadas como eje transversal de análisis y elaboración de los estudios de caso.

A partir de estas informaciones y de la revisión de literatura se elaboró un informe de caso de Mapeo de APS por país, que se encuentra en su versión completa disponible en el sitio del Isags (http://www.isags-unasur.org).

Cuadro 1. Matriz para el análisis de la APS en los países: dimensiones y componentes

\begin{tabular}{ll}
\hline Dimensiones & Componentes \\
\hline Conducción de la APS & Concepciones de Atención Primaria de Salud en las políticas de salud \\
& $\begin{array}{l}\text { Atribuciones de las esferas gubernamentales y/o seguros sociales en la implementación de } \\
\text { la política de APS }\end{array}$ \\
Financiamiento de la & Participación de las esferas gubernamentales en el financiamiento de la APS \\
APS & Existencia de copagos en la APS \\
& Mecanismos para transferencias financieras entre esferas gubernamentales para la APS \\
Prestación y & Tipos de unidades de salud que brindan APS \\
organización de la APS & Principales acciones/servicios ofertados en APS \\
& Composición y organización de los equipos de APS \\
& Territorialización y adscripción poblacional para la APS \\
& Número de personas por equipo \\
& Garantías de acceso y definición de metas de tiempo máximo para consulta en atención \\
primaria & Cobertura poblacional estimada por los servicios de APS en el nuevo modelo asistencial \\
Organización del sistema de salud en niveles de atención \\
Coordinación de los \\
cuidados e integración la APS en la red de \\
servicios
\end{tabular}




\begin{tabular}{ll}
\hline Fuerza de trabajo en APS & Disponibilidad de profesionales para la APS (médicos/as y enfermeros/as) \\
& Denominación de la especialidad médica para APS \\
& Regulación de las relaciones laborales en APS (vínculos de trabajo, formas de \\
& remuneración, carrera funcionaria para APS \\
& Formación y atribuciones de los promotores/agentes de salud \\
& Estrategias de formación de la fuerza de trabajo para la APS (pregrado; posgrado y \\
& desarrollo profesional continuo) \\
& Interacción con otros sectores de políticas públicas en nivel nacional para hacer frente a los \\
& determinantes sociales \\
Actividades & Atribuciones de los/as profesionales/trabajadores/as del equipo APS en el desarrollo de \\
intersectoriales & acciones intersectoriales/ comunitarias \\
& Acción comunitaria de los equipos de APS (relaciones de los equipos APS con otros \\
& sectores de políticas públicas u organizaciones en el territorio; realización por los equipos \\
& de APS de diagnóstico de los problemas en su territorio y planificación de intervenciones \\
& locales) \\
& Formas de representación y participación de los/as usuarios/as en APS \\
Participación social en & Concepciones de interculturalidad \\
Interculturalidad en APS & Articulación de los servicios de APS con agentes de medicina tradicional de los pueblos \\
originarios y campesinos
\end{tabular}

Fuente: Elaboración propia

Este artículo sintetiza los resultados del mapeo realizado en los 12 países de América del Sur según las principales dimensiones de la matriz, trazando paralelos, contrastando diferencias, destacando similitudes e identificando sus condicionantes, en especial aquellos concernientes a características del sistema de protección social y de salud de los países, que influencian las formas de concebir e implementar la APS.

No se trata de una comparación stricto sensu, pues se entiende que la configuración actual de los sistemas de salud y de la APS en cada país está condicionada por trayectorias históricas, políticas y económicas distintas y que las comparaciones valorativas de desempeño de los sistemas de salud con producción de índices sintéticos y ordenamiento de más y menos son inapropiadas para el análisis (OLIVER, 2012). La comparación en nuestro enfoque no pretende, por lo tanto, establecer una valoración de más o menos; de mejor o peor, sino contribuir con un enfoque más analítico que permita elucidar los principales desafíos para la concreción de una atención primaria integral a la salud en nuestros países.
Para contextualizar el análisis de la APS, el artículo comienza con una breve síntesis sobre las características de los sistemas de salud en Suramérica, identificando los principales segmentos de cobertura poblacional e informando el subsistema al cual se refiere el análisis de la APS. A continuación se describen y analizan cada una de las dimensiones de APS y sus principales componentes.

\section{El contexto: características de los sistemas de salud en Suramérica}

En Suramérica, a diferencia de los países europeos, la universalización de la protección social en salud no se completó. Vistas desde afuera, tradicionalmente, las principales características que se destacan en los sistemas de salud de América Latina son la segmentación de la cobertura, la fragmentación organizacional y la privatización en la financiación y en la prestación de servicios de salud (GIOVANELLA ET AL., 2012). 
Los modelos clásicos europeos de seguro social bismarckiano con base en cotizaciones sociales obligatorias de empleados y empleadores y el modelo beveridgiano de servicio nacional de salud, de acceso universal con base en la ciudadanía y financiado con recursos fiscales, influenciaron las políticas de salud de la región, pero no fueron plenamente implementados.

En la mayor parte de los países de Suramérica persiste una importante segmentación de la protección social en salud con la presencia de diversos subsistemas con distintas reglas de financiación, afiliación, acceso a prestaciones y red de servicios, responsables por la atención en salud de diferentes grupos poblacionales de acuerdo a su estatus laboral, pertenencia social y capacidad de pago. Se pueden identificar cuatro segmentos principales (cuadro 2): i) un segmento de seguro social que cubre grupos de ingresos medios incluidos en el mercado formal de trabajo (20\% a $40 \%$ de la población); ii) un sistema público de salud con cobertura parcial de la población de menores ingresos por prestación pública directa; iii) una cobertura de paquete selectivo por seguros públicos focalizados en grupos específicos, como el materno infantil, adultos mayores o debajo de determinado nivel de ingreso, implementados a partir de los años 1990; iv) seguros privados de salud (medicina prepaga) para cobertura de grupos de mayor renta; además del pago de bolsillo, que afecta casi todos los grupos y de la persistencia de una importante exclusión en salud en algunos países.
Para cada segmento, los servicios cubiertos y los prestadores son distintos y conforman subsistemas de salud de distintas clases con importantes inequidades. La otra cara de la segmentación es la fragmentación, con ausencia de coordinación entre las instituciones públicas, de seguridad social y privadas, y la existencia de muchas entidades no coordinadas en el interior de cada subsistema. La fragmentación conduce a la discontinuidad de los cuidados y a la ineficiencia.

Desde la perspectiva de la financiación, la cobertura en salud en Suramérica es fuertemente privatizada. En siete de los 12 países de Suramérica la participación de los gastos públicos en el total de gastos en salud no alcanza al $60 \%$. No obstante, se observa un aumento de la participación pública en los gastos en salud en seis países para el periodo 2000-2010, señalando una tendencia de mejora en la cobertura pública en la Región (cuadro 2).

Por otra parte, la participación de organizaciones privadas en la prestación de servicios de salud es alta en muchos países, como por ejemplo: en Argentina 68\% de los establecimientos con internación son privados; en Brasil 65\% de las camas y $86 \%$ de los tomógrafos son privados (GIOVANELLA, 2013). La elevada participación privada en la prestación dificulta el acceso para los usuarios del sistema público y la integración de la red asistencial, lo que exige una gran capacidad de regulación de los gobiernos, aún poco desarrollada. 
Cuadro 2. Segmentación de los sistemas de salud en Suramérica: cobertura poblacional por segmento, 2010, y gastos totales en salud en \% del PIB y gasto público como \% del gasto total en salud en los países suramericanos, 2000 y 2010

\begin{tabular}{|c|c|c|c|c|c|c|c|}
\hline \multirow[t]{2}{*}{ Países } & \multirow{2}{*}{$\begin{array}{l}\text { Seguros sociales } \\
\text { (trabajadores } \\
\text { mercado formal) }\end{array}$} & \multirow[t]{2}{*}{$\begin{array}{l}\text { Seguros públicos } \\
\text { focalizados }\end{array}$} & \multirow{2}{*}{$\begin{array}{l}\text { Sistema público/ } \\
\text { Ministerio de } \\
\text { Salud/ esferas } \\
\text { subnacionales }\end{array}$} & \multirow{2}{*}{$\begin{array}{c}\text { Seguros } \\
\text { privados y } \\
\text { medicina } \\
\text { prepaga }\end{array}$} & \multirow{2}{*}{$\begin{array}{c}\text { Gastos totales } \\
\text { en salud como } \\
\% \text { del PIB } \\
2010\end{array}$} & \multicolumn{2}{|c|}{$\begin{array}{l}\text { Gasto público como \% del } \\
\text { gasto total en salud }\end{array}$} \\
\hline & & & & & & 2000 & 2010 \\
\hline Argentina & ++++ & + & ++ & + & 8,3 & 53,9 & 64,4 \\
\hline Bolivia & + & +++ & + & - & 5,5 & 60,1 & 66,2 \\
\hline Brasil & - & - & +++++ & ++ & 9,0 & 40,3 & 47,0 \\
\hline Chile & +++++ & - & - & ++ & 7,4 & 52,1 & 59,5 \\
\hline Colombia & +++ & ++++ & - & + & 6,5 & 79,3 & 74,6 \\
\hline Ecuador & ++ & + & +++ & + & 7,9 & 31,2 & 40,2 \\
\hline Guyana & + & + & + & + & 4,2 & 84,7 & 79,5 \\
\hline Paraguay & + & - & +++ & + & 9,6 & 39,9 & 34,4 \\
\hline Perú & ++ & +++ & + & + & 4,9 & 58,7 & 56,2 \\
\hline Suriname & ++ & ++ & - & + & 5,3 & 53,4 & 51,7 \\
\hline Uruguay & ++++ & - & ++ & + & 8,1 & 54,6 & 65,3 \\
\hline Venezuela & ++ & - & +++ & + & 5,3 & 41,5 & 38,8 \\
\hline
\end{tabular}

Fuente: Elaboración propria

Notas: $+\leq 15 \% ;++=16-30 \% ;+++=31-50 \% ;++++=51-70 \% ;+++++\geq 71 \%$

Los procesos de reformas neoliberales en salud que acompañaron los programas de ajuste estructural de los años 1980 y 1990 acentuaron las características de segmentación, fragmentación y privatización de los sistemas de salud y la selectividad de la atención primaria en Suramérica. De esta forma, profundizaron las desigualdades sociales constituyendo ciudadanos de primera, segunda y tercera clase que acceden, o no, a diferentes paquetes y redes de servicios diferenciados conforme a su capacidad de pago. Este legado institucional obstaculiza sobremanera las reformas de los gobiernos progresistas actuales en pro de la garantía del derecho a la salud y de la construcción de sistemas públicos de salud universales.

\section{Concepciones de APS en las políticas de salud}

Los documentos de las políticas de salud actuales de los países de Suramérica destacan la APS como estrategia para la garantía de la atención integral y la equidad en salud. La referencia a la Declaración de Alma-Ata es explicitada en las políticas o programas en los 12 países, así como el proceso de renovación, señalado en diez países (excepto Guyana y Paraguay).

Las políticas nacionales incorporan con diferentes énfasis los tres componentes principales de la Declaración de Alma-Ata que concibe la APS como estrategia: i) como atención esencial, parte integrante de un sistema nacional de cuidados de salud, del cual es el núcleo principal, primer nivel de contacto $\mathrm{y}$ elemento de un proceso permanente de atención sanitaria; ii) la salud como indisociable del desarrollo económico-social de una sociedad, lo que implica incidir en los determinantes sociales; y iii) el fomento a la participación social.

En este proceso de revitalización de la APS - denominado en algunos países APS renovada, por influencia de OPS -, siete países están en proceso de gestión e implementación de nuevos modelos de atención (cuadro 3). Estos nuevos modelos tienen componentes comunes: el enfoque familiar, la mención a la atención integral, el enfoque 
comunitario con equipos multiprofesionales con población y territorios definidos (Bolivia, Brasil, Chile, Ecuador, Paraguay, Perú y Venezuela). Venezuela, Ecuador y Bolivia comparten el énfasis en el enfoque intercultural con respeto y asimilación de los saberes y prácticas en salud de los pueblos originarios expresados en la concepción del 'buen vivir'. Estos países comparten también la prioridad de la promoción de la salud entendida como la acción intersectorial sobre sus determinantes sociales.

Cuadro 3. Concepción/definición de Atención Primaria de Salud en las políticas de salud actuales, países suramericanos

\begin{tabular}{|c|c|}
\hline Países & APS en la política actual \\
\hline $\begin{array}{l}\text { Argentina } \\
(2004)\end{array}$ & $\begin{array}{l}\text { APS es la estrategia que concibe integralmente los problemas de salud-enfermedad-atención de las personas y del conjunto social, a } \\
\text { través de la integración de la asistencia, la prevención de enfermedades, la promoción de la salud y la rehabilitación. } \\
\text { Diversos programas federales se vinculan directamente con la estrategia de APS (Programa Médicos Comunitarios, Remediar + Redes, } \\
\text { Plan Nacer). }\end{array}$ \\
\hline $\begin{array}{l}\text { Bolivia } \\
(2008)\end{array}$ & $\begin{array}{l}\text { No se emplea el término APS en las normas. El 'Modelo de Salud Familiar Comunitario Intercultural (Safci)' con sus principios de } \\
\text { participación comunitaria, intersectorialidad, interculturalidad e integralidad contempla los elementos de APS. El modelo Safci se } \\
\text { define como el conjunto de acciones que facilitan el desarrollo de procesos de promoción de la salud, prevención y tratamiento de la } \\
\text { enfermedad y rehabilitación de manera eficaz, eficiente y oportuna en el marco de la horizontalidad, integralidad e interculturalidad, de } \\
\text { tal manera que las políticas de salud se presentan y articulan con las personas, familias y la comunidad o barrio. }\end{array}$ \\
\hline $\begin{array}{l}\text { Brasil } \\
(2006, \\
2011)\end{array}$ & $\begin{array}{l}\text { La atención básica se caracteriza por un conjunto de acciones de salud en el ámbito individual y colectivo que abarca la promoción y la } \\
\text { protección de la salud, la prevención de enfermedades, el diagnóstico, el tratamiento, la rehabilitación, la reducción de daños y la manutención } \\
\text { de la salud con el objetivo de desarrollar una atención integral que tenga impacto en la situación de salud, en la autonomía de las personas y } \\
\text { en los determinantes y condicionantes de salud de las colectividades. } \\
\text { La 'Estrategia Salud de la Familia (ESF)', con sus equipos multiprofesionales de base territorial, es el principal modelo de atención en APS. }\end{array}$ \\
\hline $\begin{array}{l}\text { Chile } \\
(2005, \\
2013)\end{array}$ & $\begin{array}{l}\text { El nuevo 'Modelo de Atención Integral de Salud, Familiar y Comunitaria' busca asegurar una distribución más equitativa de los } \\
\text { recursos de salud para otorgar una asistencia sanitaria esencial basada en métodos y tecnologías prácticos, científicamente fundados } \\
\text { y socialmente aceptables, puesta al alcance de todos los individuos y familias de la comunidad mediante su plena participación } \\
\text { y a un coste que la comunidad y el país puedan soportar en todas y cada una de las etapas de su desarrollo, con un espíritu de } \\
\text { autorresponsabilidad y autodeterminación. }\end{array}$ \\
\hline
\end{tabular}

Colombia La APS es la estrategia de coordinación intersectorial que permite la atención integral e integrada, desde la salud pública, la promoción (2011) de la salud, la prevención de la enfermedad, el diagnóstico, el tratamiento y la rehabilitación del paciente en todos los niveles de complejidad a fin de garantizar un mayor nivel de bienestar en los usuarios, sin perjuicio de las competencias legales de cada uno de los actores del Sistema General de Seguridad Social en Salud.

La Ley 1438 de 2011 introdujo la Atención Primaria de Salud como estrategia nacional, pero aún no fue implementada, no existe un modelo único de APS y las experiencias son de base local.

Ecuador La Constitución de 2008 define la Atención Primaria de Salud como base del sistema en su Art. 360: "El sistema garantizará, a través (2008, 2012) de las instituciones que lo conforman, la promoción de la salud, prevención y atención integral, familiar y comunitaria, 'con base en la Atención Primaria de Salud'; articulará los diferentes niveles de atención; y promoverá la complementariedad con las medicinas ancestrales y alternativas".

El 'Modelo de Atención Integral en Salud Familiar Comunitaria e Intercultural (Mais-FCI)' incorpora la estrategia de la APS renovada. ES el conjunto de estrategias que organizan el sistema Nacional de Salud, para responder a las necesidades de salud de las personas, las familias, las comunidades y el entorno, permitiendo la integralidad en los niveles de atención de la red de salud.

Guyana El documento Visión de Salud 2020 reafirma el compromiso con la APS y la importancia de enfrentar los determinantes sociales de la

$(2013$,

2010)

Paraguay Se busca incrementar el acceso para la población excluida a través de 'equipos y unidades de salud de la familia' asignados a territorios (2008) definidos

La APS en la gestión actual es entendida como: una estrategia que concibe integralmente el proceso salud-enfermedad y de atención a las personas y comunidades, considerando las diferentes etapas de la vida. Provee servicios de salud y enfrenta las causas últimas (sociales, económicas, políticas y ambientales) de la ausencia de salud. 


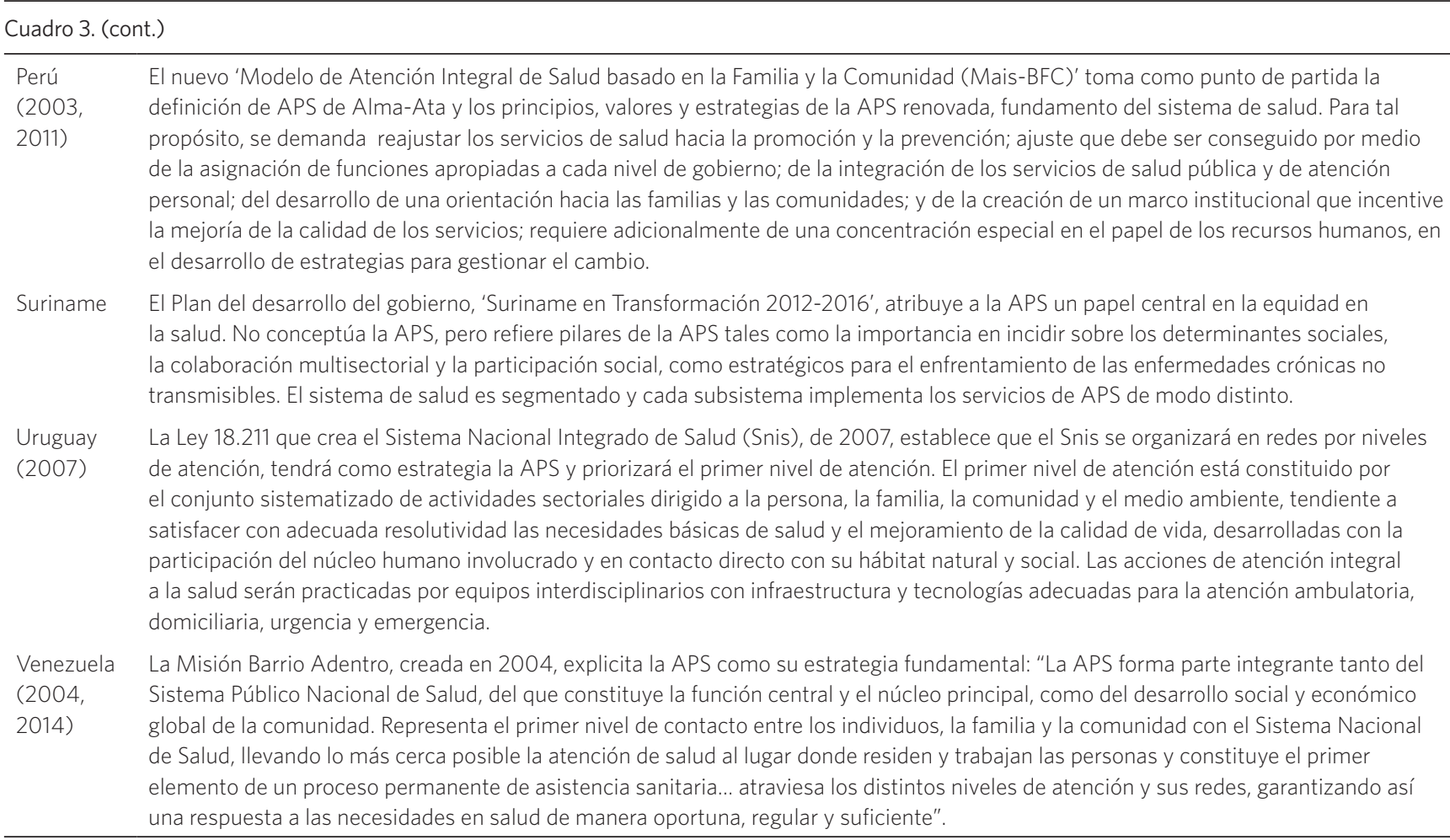

\section{Atribuciones de las esferas gubernamentales y/o seguros sociales en la formulación, financiación e implementación de la política de APS}

La modalidad de protección social en salud y la organización territorial, política y administrativa de los países condicionan la distribución de atribuciones entre las esferas gubernamentales y/o seguros sociales en la formulación e implementación de la política de APS.

En general, la esfera responsable por la formulación de la política de APS es el nivel nacional, el Ministerio de Salud. En Argentina, con su organización federal y autonomía de las provincias en materia de salud, esta atribución es principalmente provincial. En Colombia, el sistema de seguro social en salud, con su enfoque individual, no incorporó un modelo de atención con base en la APS. La formulación e implementación de programas de APS permaneció como una iniciativa de gobiernos locales, con experiencias diversificadas. En 2011, la legislación nacional definió una política de APS, aún en fase de reglamentación (cuadro 4).

Las fuentes de financiación de la APS son las mismas que las de la financiación del sistema de salud en general. Considerando solamente la financiación pública, en la mayor parte de los países la responsabilidad por la financiación de la APS es del nivel nacional. En Brasil, esta responsabilidad es compartida con los municipios y en Argentina con las provincias. En Bolivia y Perú hay participación de los seguros focalizados en los más pobres o en grupos específicos. En Colombia, las iniciativas en APS y su financiación son municipales y la atención individual de primer nivel es financiada por el Sistema General de Seguridad Social 
a través de las Empresas Promotoras de Salud (EPS) del régimen contributivo y del régimen subsidiado.

Como consecuencia de la segmentación, cotizaciones para los seguros sociales de salud para trabajadores del mercado formal financian la atención individual de primer nivel para este grupo poblacional en 11 países, excepto Brasil (cuadro 2).

Con diferentes grados de descentralización de sus sistemas de salud, la responsabilidad por la prestación de servicios de primer nivel es diferenciada, prevaleciendo la esfera municipal en Brasil, Chile y Colombia. Las provincias o departamentos tienen un importante rol en la prestación de servicios de primer nivel en Argentina, Ecuador y Bolivia. El Ministerio de Salud permanece como el principal prestador en Uruguay, Paraguay Guyana y Venezuela, responsabilidad compartida con diversas ONG en Suriname (cuadro 4).

Las transferencias financieras para APS desde el nivel nacional a las esferas subnacionales se procesan de modos muy diversos. Los Ministerios de Salud transfieren recursos financieros por programas específicos incentivados por el gobierno nacional (Argentina, Ecuador, Guyana, Venezuela) o, en los sistemas públicos más descentralizados, como Brasil y
Chile, por cápitas específicas para APS. En los sistemas públicos más centralizados, la ejecución financiera es del nivel central, como en Paraguay, Uruguay y Suriname. En los modelos de protección en salud con base a seguros no hay transferencias financieras desde el Ministerio de Salud hacia las esferas subnacionales para la APS. En estos casos (y/o en estos segmentos) las transferencias son para los efectores del seguro, como en Bolivia, Colombia y Uruguay. Esto hace que a veces se confundan con las formas de pago al proveedor final, pues las esferas gubernamentales son tratadas como proveedores por los efectores de los seguros.

En la mayor parte de los países, el acceso a la APS es gratuito y no hay copagos en los servicios públicos de primer nivel. Los copagos introducidos en la década de 1990 fueron abolidos con los gobiernos progresistas en Venezuela, Ecuador y Paraguay. En Bolivia y Perú hay cobro de las atenciones en los servicios públicos de salud, con excepción de los grupos poblacionales cubiertos por los seguros públicos focalizados (cuadro 4).

No obstante, en la mayor parte de los países los gastos privados en salud son elevados (cuadro 2) y subsisten gastos de bolsillo de los hogares por pagos de medicamentos y algunos servicios de primer nivel.

\begin{tabular}{|c|c|c|c|c|c|c|}
\hline Países & $\begin{array}{c}\text { Organización } \\
\text { político- } \\
\text { administrativa del } \\
\text { país }\end{array}$ & $\begin{array}{l}\text { Principal } \\
\text { responsable por } \\
\text { la formulación de } \\
\text { la política de APS }\end{array}$ & $\begin{array}{c}\text { Principal } \\
\text { responsable por } \\
\text { el financiamiento } \\
\text { de APS }\end{array}$ & $\begin{array}{c}\text { Principal } \\
\text { responsable por } \\
\text { la prestación de } \\
\text { servicios de primer } \\
\text { nivel }\end{array}$ & $\begin{array}{c}\text { Mecanismos de } \\
\text { transferencias } \\
\text { financieras específicas } \\
\text { para APS desde el } \\
\text { nivel nacional hasta las } \\
\text { esferas subnacionales }\end{array}$ & $\begin{array}{l}\text { Copagos y pagos } \\
\text { en servicios } \\
\text { públicos de } \\
\text { primer nivel }\end{array}$ \\
\hline Argentina & $\begin{array}{l}\text { República Federal } \\
23 \text { provincias }+ \\
\text { CABA } \\
2.200 \text { municipios } \\
\text { Constitución } 1994\end{array}$ & $\begin{array}{l}\text { Ministerios de } \\
\text { Salud Provinciales } \\
\text { y Ministerio } \\
\text { de Salud de la } \\
\text { Nación }\end{array}$ & $\begin{array}{l}\text { MINSAL y } \\
\text { Provincias } \\
\text { (Obras Sociales: } \\
\text { atención individual } \\
\text { de primer nivel) }\end{array}$ & $\begin{array}{l}\text { Provincias y } \\
\text { Municipios }\end{array}$ & Por programa específico & No hay \\
\hline Bolivia & $\begin{array}{l}\text { Estado Plurinacional } \\
9 \text { Departamentos } \\
337 \text { municipios } \\
\text { Constitución } 2009\end{array}$ & $\begin{array}{l}\text { Ministerio de } \\
\text { Salud }\end{array}$ & $\begin{array}{l}\text { Departamentos } \\
\text { Ministerio de } \\
\text { Salud } \\
\text { Municipios } \\
\text { Seguros } \\
\text { focalizados SUMI } \\
\text { y SSPAM }\end{array}$ & Departamentos & $\begin{array}{l}\text { Per cápita a municipios } \\
\text { para el seguro público } \\
\text { focalizado para niños, } \\
\text { embarazadas y adultos } \\
\text { mayores. Paquetes } \\
\text { presupuestarios para } \\
\text { todos los demás servicios. }\end{array}$ & $\begin{array}{l}\text { Pago en servicios } \\
\text { públicos, } \\
\text { excepto para } \\
\text { los asegurados } \\
\text { de los seguros } \\
\text { focalizados }\end{array}$ \\
\hline
\end{tabular}




\begin{tabular}{|c|c|c|c|c|c|c|}
\hline \multicolumn{7}{|c|}{ Cuadro 4. (cont.) } \\
\hline Brasil & $\begin{array}{l}\text { República Federativa } \\
\text { DF }+26 \text { estados } \\
5.570 \text { municipios } \\
\text { Constitución } 1988\end{array}$ & $\begin{array}{l}\text { Ministerio de } \\
\text { Salud }\end{array}$ & $\begin{array}{l}\text { Ministerio de } \\
\text { Salud y } \\
\text { Municipios }\end{array}$ & Municipios & $\begin{array}{l}\text { Per cápita específico } \\
\text { para APS: Piso de } \\
\text { Atención Básica; Piso de } \\
\text { Atención Básica Variable } \\
\text { por equipo de ESF y } \\
\text { desempeño }\end{array}$ & No hay \\
\hline Chile & $\begin{array}{l}\text { Estado Unitario } \\
15 \text { regiones } \\
53 \text { provincias y } 346 \\
\text { comunas } \\
\text { Constitución } 1980\end{array}$ & $\begin{array}{l}\text { Ministerio de } \\
\text { Salud }\end{array}$ & $\begin{array}{l}\text { Ministerio de } \\
\text { Salud (Fonasa) y } \\
\text { municipios }\end{array}$ & $\begin{array}{l}\text { Municipios y } \\
\text { Servicios de Salud }\end{array}$ & $\begin{array}{l}\text { Per cápita específico para } \\
\text { APS y aportes adicionales } \\
\text { del estado }\end{array}$ & $\begin{array}{l}\text { No hay para los } \\
\text { grupos A y B del } \\
\text { Fonasa } \\
\text { (copagos para } \\
\text { asegurados de } \\
\text { los tramos C y D } \\
\text { por las garantías } \\
\text { y prestaciones } \\
\text { que tengan } \\
\text { copagos en el } \\
\text { AUGE) }\end{array}$ \\
\hline Colombia & $\begin{array}{l}\text { República Unitaria } \\
\text { DC }+32 \\
\text { departamentos } \\
1.102 \text { municipios } \\
\text { Constitución } 1991\end{array}$ & $\begin{array}{l}\text { Municipios } \\
\text { (política } \\
\text { nacional en } \\
\text { reglamentación) }\end{array}$ & $\begin{array}{l}\text { Presupuesto de la } \\
\text { nación } \\
\text { Municipios } \\
\text { SGSS - EPS } \\
\text { régimen } \\
\text { subsidiado y } \\
\text { contributivo } \\
\text { (individual) }\end{array}$ & $\begin{array}{l}\text { Municipios y } \\
\text { Departamentos; } \\
\text { EPS régimen } \\
\text { subsidiado y } \\
\text { contributivo; } \\
\text { contratan servicios } \\
\text { públicos o privados }\end{array}$ & $\begin{array}{l}\text { Sistema general de } \\
\text { participaciones } \\
\text { Unidades de pago per } \\
\text { cápita }\end{array}$ & $\begin{array}{l}\text { No hay copagos } \\
\text { para promoción } \\
\text { y prevención, } \\
\text { atención } \\
\text { materno-infantil, } \\
\text { enfermedades } \\
\text { transmisibles, } \\
\text { patologías de } \\
\text { alto costo y } \\
\text { atención inicial } \\
\text { de urgencias }\end{array}$ \\
\hline Ecuador & $\begin{array}{l}\text { Estado Unitario } \\
\text { Plurinacional } \\
24 \text { provincias } \\
210 \text { cantones } \\
1.000 \text { parroquias } \\
\text { Constitución } 2008\end{array}$ & $\begin{array}{l}\text { Ministerio de } \\
\text { Salud Pública }\end{array}$ & $\begin{array}{l}\text { Ministerio de la } \\
\text { Salud Pública y } \\
\text { Provincias }\end{array}$ & Provincias & $\begin{array}{l}\text { Por presupuesto global y } \\
\text { programa específico }\end{array}$ & $\begin{array}{l}\text { No hay } \\
\text { (abolidos en } \\
\text { 2008) }\end{array}$ \\
\hline Guyana & $\begin{array}{l}\text { República } \\
\text { Cooperativa } \\
10 \text { regiones } \\
27 \text { consejos de } \\
\text { barrios } \\
\text { Constitución } 1980\end{array}$ & $\begin{array}{l}\text { Ministerio de } \\
\text { Salud }\end{array}$ & $\begin{array}{l}\text { Ministerio de la } \\
\text { Salud y donaciones } \\
\text { externas }\end{array}$ & $\begin{array}{l}\text { Servicios regionales } \\
\text { del Ministerio de } \\
\text { Salud } \\
\text { ONG } \\
\text { internacionales }\end{array}$ & Por programa específico & No hay \\
\hline Paraguay & $\begin{array}{l}\text { República } \\
\text { Democrática } \\
\text { Representativa } \\
\text { Capital + } 17 \\
\text { departamentos } \\
\text { Constitución } 1992\end{array}$ & $\begin{array}{l}\text { Ministerio de } \\
\text { Salud y Bienestar } \\
\text { Social }\end{array}$ & $\begin{array}{l}\text { Ministerio de } \\
\text { Salud y Bienestar } \\
\text { Social }\end{array}$ & $\begin{array}{l}\text { Ministerio de Salud } \\
\text { y Bienestar Social y } \\
\text { Municipios }\end{array}$ & No hay & $\begin{array}{l}\text { No hay } \\
\text { (abolidos en } \\
\text { 2008) }\end{array}$ \\
\hline Perú & $\begin{array}{l}\text { República } \\
\text { Democrática } \\
24 \text { departamentos } \\
195 \text { provincias } \\
1.837 \text { municipios } \\
\text { Constitución } 1993\end{array}$ & $\begin{array}{l}\text { Ministerio de } \\
\text { Salud }\end{array}$ & $\begin{array}{l}\text { Ministerio de la } \\
\text { Salud } \\
\text { Seguro focalizado: } \\
\text { SIS } \\
\text { Seguro Social: } \\
\text { EsSalud }\end{array}$ & $\begin{array}{l}\text { Gobiernos locales } \\
\text { Administradoras } \\
\text { de Fondos del } \\
\text { Aseguramiento } \\
\text { en Salud (lafas) } \\
\text { contratan servicios } \\
\text { públicos o privados }\end{array}$ & $\begin{array}{l}\text { No hay } \\
\text { (el seguro focalizado } \\
\text { SIS paga a los } \\
\text { establecimientos montos } \\
\text { fijos cuatrimestrales y un } \\
\text { monto variable trimestral) }\end{array}$ & $\begin{array}{l}\text { Pago en los } \\
\text { servicios } \\
\text { públicos para los } \\
\text { medicamentos } \\
\text { para } \\
\text { población no } \\
\text { asegurada a } \\
\text { EsSalud o SIS }\end{array}$ \\
\hline
\end{tabular}




\begin{tabular}{|c|c|c|c|c|c|c|}
\hline \multicolumn{7}{|c|}{ Cuadro 4. (cont.) } \\
\hline Suriname & $\begin{array}{l}\text { República } \\
\text { Democrática } \\
10 \text { distritos } \\
62 \text { regiones } \\
\text { Constitución } 1987\end{array}$ & $\begin{array}{l}\text { Ministerio de } \\
\text { Salud }\end{array}$ & $\begin{array}{l}\text { Ministerio de } \\
\text { Salud }\end{array}$ & $\begin{array}{l}\text { Ministerio de Salud } \\
\text { ONG: Misión } \\
\text { Médica }\end{array}$ & No hay & $\begin{array}{l}\text { Copago para } \\
\text { medicamentos. } \\
\text { Para la población } \\
\text { atendida por la } \\
\text { Misión Médica } \\
\text { no hay copago }\end{array}$ \\
\hline Uruguay & $\begin{array}{l}\text { República Estado } \\
\text { Unitario } \\
19 \text { departamentos } \\
\text { Constitución } 1997\end{array}$ & $\begin{array}{l}\text { Ministerio de } \\
\text { Salud Pública }\end{array}$ & $\begin{array}{l}\text { Ministerio de } \\
\text { Salud Pública. } \\
\text { Administración de } \\
\text { Servicios de Salud } \\
\text { del Estado (ASSE) }\end{array}$ & $\begin{array}{l}\text { Administración de } \\
\text { Servicios de Salud } \\
\text { del Estado (ASSE) } \\
\text { Intendencias } \\
\text { Municipales. } \\
\text { Seguros sociales: } \\
\text { servicios } \\
\text { ambulatorios del } \\
\text { sector privado } \\
\text { regulado }\end{array}$ & $\begin{array}{l}\text { No hay } \\
\text { (transferencias de } \\
\text { Fonasa a los efectores: } \\
\text { per cápita ajustada al } \\
\text { riesgo por edad y sexo + } \\
\text { cumplimiento de metas } \\
\text { asistenciales) }\end{array}$ & $\begin{array}{l}\text { No hay } \\
\text { (copagos } \\
\text { regulados en } \\
\text { los efectores } \\
\text { privados para el } \\
\text { seguro social) }\end{array}$ \\
\hline Venezuela & $\begin{array}{l}\text { República Bolivariana } \\
23 \text { estados + DC } \\
335 \text { municipios } \\
1123 \text { parroquias } \\
\text { Constitución } 1999\end{array}$ & $\begin{array}{l}\text { Ministerio del } \\
\text { Poder Popular } \\
\text { para la Salud }\end{array}$ & $\begin{array}{l}\text { Ministerio del } \\
\text { Poder Popular para } \\
\text { la Salud }\end{array}$ & $\begin{array}{l}\text { Municipios y } \\
\text { Ministerio del } \\
\text { Poder Popular para } \\
\text { la Salud }\end{array}$ & $\begin{array}{l}\text { Paquete presupuestario y } \\
\text { por programa específico }\end{array}$ & $\begin{array}{l}\text { No hay } \\
\text { (abolidos en } \\
\text { 1998) }\end{array}$ \\
\hline
\end{tabular}

Fuentes: Elaboración propria

Sumi - Seguro Universal de Maternidad y Niñez; SSPAM - Seguro de Salud del Adulto Mayor; SGSSS - Sistema General de Seguridad Social en Salud; SIS - Seguro Integral de Salud focalizado en los más pobres; EsSalud - Seguro Social de Salud

\section{Prestación y organización de la APS}

La prestación se caracteriza por una composición de los equipos de APS diversificada entre los países y diferenciada conforme el tipo de unidad de salud. En los 12 países, los principales tipos de unidades de salud que brindan la APS son los Centros de Salud y los Puestos de Salud, estos últimos, sobre todo, en el área rural, donde trabajan auxiliares o técnicos de enfermería o Agentes Comunitarios de Salud (ACS). En Chile y Venezuela, hospitales de baja complejidad y ambulatorios también forman parte de la APS. En Colombia, la APS es prestada en Instituciones Prestadoras de Servicios de Salud de Primer Nivel, que son elegidas de acuerdo con la inserción del usuario en el Seguro Social de Salud. En los Centros de Salud, el/la médico/a general y el/la enfermero/a están presentes en los equipos todos los países. Uruguay cuenta también con pediatra, ginecólogo/a y partero/a. El/la auxiliar y/o técnico/a de enfermería también forma parte de los equipos en la mayor parte de los países.

Como parte de las reformas de los modelos de atención en salud, diversos países incorporaron el/la Agente Comunitario/a de Salud o Promotor/a de Salud como trabajador/a remunerado/a o voluntario/a de los equipos básicos de APS, con distintas denominaciones y en el rol de enlace de la comunidad con el establecimiento de salud y ejecución de tareas extramurales y comunitarias. En la mayoría de los casos, los/as Agentes/Promotores/ as son personas sin formación en salud que viven en las áreas de los servicios de APS. En Ecuador, Guyana y Suriname, asistentes de salud generalistas con formación técnica, actúan en los equipos de APS, principalmente en áreas rurales (cuadro 5).

La territorialización de los servicios de la APS con adscripción poblacional y población asignada por equipo, con responsabilidad de los equipos por poblaciones definidas, se observa en ocho países. El número de personas 
asignadas por equipo varía tanto en el propio país como entre los países, siendo 1.250 usuarios por equipo en Venezuela, 3.000 en Brasil, 4.000 en Ecuador, hasta 5.000 en Chile y Paraguay, en promedio (cuadro 5). El número de personas asignadas por equipo de APS sufre variaciones en función de las características de los territorios, como la pertenencia a áreas rurales y/o de mayor vulnerabilidad social (RÍOS, 2014B; ALMEIDA, 2014; VEGA ROMERO; ACOSTA RAMÍREZ, 2014A; TEJERINA SILVA, 2014B, 2014C).

La cobertura poblacional por los nuevos modelos de atención con énfasis en la APS renovada, en gestión e implementación en siete países (Bolivia, Brasil, Chile, Ecuador, Paraguay, Perú y Venezuela), es variable y depende del ritmo de implementación de los cambios. En Brasil, 62\% de la población está cubierta por la 'Estrategia Salud de la Familia'; en Paraguay 36\% por 'Unidades de Salud de la Familia'; en Venezuela cerca de $60 \%$ por los equipos de APS de la 'Misión Barrio Adentro'. En Bolivia y Perú no hay datos precisos sobre la población cubierta por el modelo de 'Salud Familiar Comunitario Intercultural', en el primero, y por los 'Equipos de Atención Integral en Salud a Poblaciones Excluidas y Dispersas' y el 'Modelo de Atención Integral de Salud Familiar y Comunitario', en el segundo (RíOS, 2014B; ACOSTA RAMÍREZ; VEGA ROMERO, 2014; ALMEIDA, 2014; VEGA ROMERO; ACOSTA RAMÍREZ, 2014A; TEJERINA SILVA, 2014A, 2014B, 2014C).

Cuadro 5. Prestación de servicios de primer nivel y organización de la Atención Primaria de Salud en los países suramericanos

\begin{tabular}{|c|c|c|c|c|c|}
\hline Países & $\begin{array}{c}\text { Principales tipos de } \\
\text { unidades de salud que } \\
\text { brindan APS }\end{array}$ & $\begin{array}{l}\text { Composición del equipo } \\
\text { básico de APS }\end{array}$ & $\begin{array}{l}\text { Agente Comunitario } \\
\text { de Salud (ACS) } \\
\text { (pagados o } \\
\text { voluntarios) }\end{array}$ & $\begin{array}{c}\text { Territorialización y } \\
\text { adscripción poblacional } \\
\text { de los servicios de APS }\end{array}$ & $\begin{array}{c}\text { Número de } \\
\text { personas } \\
\text { asignadas por } \\
\text { equipo de APS }\end{array}$ \\
\hline Argentina & $\begin{array}{l}7.532 \text { Centros de Atención } \\
\text { Primaria de Salud (CAPS) } \\
600 \text { Centros Comunitarios } \\
\text { Integrados (CIC) }\end{array}$ & $\begin{array}{l}\text { Médicos/as, licenciados/ } \\
\text { as en enfermería, auxiliares } \\
\text { de enfermería, parteras/os, } \\
\text { agentes comunitarios/as, } \\
\text { odontólogos/as }\end{array}$ & $\begin{array}{l}\text { Agentes } \\
\text { Comunitarios/as de } \\
\text { Salud (pagados), } \\
\text { promotores/as de } \\
\text { salud }\end{array}$ & $\begin{array}{l}\text { Sí, diferenciada por } \\
\text { provincias }\end{array}$ & $3.200-4.000$ \\
\hline Bolivia & $\begin{array}{l}\text { 1.671 Centros de Salud (CS) } \\
\text { 1.604 Puestos de Salud (PS) }\end{array}$ & $\begin{array}{l}\text { CS: Médico/a general, } \\
\text { odontólogo/a, auxiliar o } \\
\text { técnico/a en enfermería } \\
\text { PS: Auxiliar o técnico/a en } \\
\text { enfermería }\end{array}$ & $\begin{array}{l}\text { Responsables } \\
\text { Populares de Salud } \\
\text { (voluntarios) }\end{array}$ & $\begin{array}{l}\text { Sí, pero la adscripción es } \\
\text { incipiente }\end{array}$ & $\begin{array}{l}\text { CS: } 1.000- \\
20.000 \\
\text { PS: } 500-1.000\end{array}$ \\
\hline Brasil & $\begin{array}{l}\text { 34.013 Unidades Básica de } \\
\text { Salud/ Centros de Salud/USF } \\
10.651 \text { Puestos de Salud } \\
\text { Total UBS activas: } 38.800 \\
\text { (2012) }\end{array}$ & $\begin{array}{l}\text { EqSF: Médico/a, } \\
\text { enfermero/a, 1-2 auxiliar/ } \\
\text { técnico/a en enfermería, } 5 \\
\text { a } 6 \text { ACS } \\
\text { Equipos de salud bucal: } \\
\text { odontólogo/a, auxiliar y/o } \\
\text { técnico/a en salud bucal }\end{array}$ & ACS (pagados) & $\begin{array}{l}\text { Sí, población asignada por } \\
\text { equipo en la Estrategia de } \\
\text { Salud de la Familia (EqSF) }\end{array}$ & $\begin{array}{l}3.000 \text { (hasta } \\
4.500 \text { ) por EqSF }\end{array}$ \\
\hline Chile & $\begin{array}{l}149 \text { Centros de Salud } \\
\text { Familiares (Cesfam) } \\
362 \text { Consultorios Generales } \\
\text { Urbanos (CGU) } \\
106 \text { Hospitales de Baja } \\
\text { Complejidad } \\
\text { 1.204 Postas de Salud Rural }\end{array}$ & $\begin{array}{l}\text { Médico/a, enfermero/a, } \\
\text { matrona, asistente social y } \\
\text { asistente administrativo/a }\end{array}$ & $\begin{array}{l}\text { Monitores/as } \\
\text { voluntarios de la } \\
\text { comunidad }\end{array}$ & $\begin{array}{l}\text { Sí, población asignada } \\
\text { por equipo, la inscripción } \\
\text { es por iniciativa de las } \\
\text { personas }\end{array}$ & $\begin{array}{l}\text { Hasta } 5.000 \\
\text { por equipo } \\
\text { multidisciplinario }\end{array}$ \\
\hline
\end{tabular}




\begin{tabular}{|c|c|c|c|c|c|}
\hline Colombia & $\begin{array}{l}996 \text { instituciones } \\
\text { prestadoras de servicios } \\
\text { de salud de primer nivel } \\
\text { públicas }\end{array}$ & $\begin{array}{l}\text { No hay una regla general } \\
\text { sobre la conformación de } \\
\text { los equipos. } \\
\text { Generalmente los equipos } \\
\text { básicos son conformados } \\
\text { por: médicos/as, } \\
\text { enfermeros/as generales, } \\
\text { promotores/as, auxiliares y } \\
\text { técnicos/as en salud. }\end{array}$ & $\begin{array}{l}\text { Promotores/as de } \\
\text { salud, caminantes } \\
\text { de la salud, agentes } \\
\text { primarios de salud } \\
\text { (voluntarios o } \\
\text { pagados) }\end{array}$ & $\begin{array}{l}\text { En algunos modelos } \\
\text { municipales } \\
\text { No hay directrices } \\
\text { nacionales sobre la } \\
\text { sectorización de centros } \\
\text { de APS y adscripción } \\
\text { poblacional. }\end{array}$ & $\begin{array}{l}\text { No hay norma } \\
\text { general, depende } \\
\text { del modelo de } \\
\text { APS de cada ente } \\
\text { territorial }\end{array}$ \\
\hline Ecuador & $\begin{array}{l}\text { 319 Puestos de Salud } \\
211 \text { Centros de Salud (A,B,C) } \\
\text { 1.387 Sub-centro de Salud } \\
\text { 1.248 Dispensarios Médicos }\end{array}$ & $\begin{array}{l}\text { Médicos/as, enfermero/a } \\
\text { y técnico/a en Atención } \\
\text { Primaria de Salud }\end{array}$ & $\begin{array}{l}\text { Técnico/a en Atención } \\
\text { Primaria de Salud } \\
\text { (Taps) (pagados) }\end{array}$ & $\begin{array}{l}\text { Sí, por circuitos y distritos } \\
\text { y adscripción poblacional } \\
\text { por dispensarización }\end{array}$ & $\begin{array}{l}4.000 \text { en áreas } \\
\text { urbanas } \\
1.500-2.500 \text { en } \\
\text { áreas rurales }\end{array}$ \\
\hline Guyana & $\begin{array}{l}201 \text { Puestos de Salud } \\
\text { (Costa: } 65 \text { / Interior: 136) } \\
229 \text { Centros de Salud } \\
\text { (Costa: } 217 / \text { Interior: 12) }\end{array}$ & $\begin{array}{l}\text { Centros de salud tipos } 1 \text { y } \\
\text { 2: médico/a, enfermero/a, } \\
\text { partero/a, Medex, asistente } \\
\text { de laboratorio, auxiliar } \\
\text { de farmacia, asistente } \\
\text { odontológico, asistente de } \\
\text { rehabilitación, asistente de } \\
\text { salud ambiental }\end{array}$ & $\begin{array}{l}\text { Técnico/a Medex y } \\
\text { ACS } \\
\text { (pagados) }\end{array}$ & No hay adscripción & - \\
\hline Paraguay & $\begin{array}{l}760 \text { Unidades de Salud de la } \\
\text { Familia }\end{array}$ & $\begin{array}{l}\text { Médico/a, licenciado/a en } \\
\text { enfermería y/u obstetricia, } \\
\text { auxiliar de enfermería y } 3 \\
\text { a } 5 \text { ACS. } \\
\text { Para cada dos ESF, un } \\
\text { equipo odontológico: } \\
\text { odontólogo/a y técnico/a } \\
\text { odontológico }\end{array}$ & $\begin{array}{l}\text { Agentes Comunitarios } \\
\text { de Salud (pagados) }\end{array}$ & $\begin{array}{l}\text { Si, población asignada por } \\
\text { equipo SF }\end{array}$ & $\begin{array}{l}3.500-5.000(u \\
800 \text { familias })\end{array}$ \\
\hline Perú & $\begin{array}{l}\text { 1.274 Establecimientos de } \\
\text { Salud del primer nivel de } \\
\text { atención } \\
153 \text { Equipos de Atención } \\
\text { Integral en Salud a } \\
\text { Poblaciones Excluidas y } \\
\text { Dispersas (Aisped) }\end{array}$ & $\begin{array}{l}\text { Médico/a, licenciado/a en } \\
\text { obstetricia, enfermero/a, } \\
\text { técnico/a en enfermería }\end{array}$ & $\begin{array}{l}\text { Agentes Comunitarios } \\
\text { de Salud o } \\
\text { promotores/as de } \\
\text { salud (voluntarios) }\end{array}$ & $\begin{array}{l}\text { Sí, los usuarios de los } \\
\text { equipos básicos son } \\
\text { registrados por área } \\
\text { de residencia en el } \\
\text { establecimiento de salud } \\
\text { de primer nivel más } \\
\text { cercano }\end{array}$ & $\begin{array}{l}500 \text { - } 800 \\
\text { familias adscritas } \\
\text { por equipo básico } \\
\text { de salud }\end{array}$ \\
\hline Suriname & $\begin{array}{l}54 \text { Puestos de Salud (Misión } \\
\text { Médica) } \\
63 \text { Clínicas de la Fundación } \\
\text { del Servicio Nacional de } \\
\text { Salud } \\
146 \text { consultorios privados GP }\end{array}$ & $\begin{array}{l}\text { Médico/a general, } \\
\text { enfermero/a, asistente de } \\
\text { salud }\end{array}$ & Asistente de salud & No hay adscripción & - \\
\hline Uruguay & $\begin{array}{l}28 \text { Centros de Salud ASSE } \\
786 \text { Consultorios, Policlínicas } \\
\text { y Puestos de Salud Rural } \\
\text { IAMC: consultas } \\
\text { externas, consultorios } \\
\text { descentralizados, servicios } \\
\text { de atención domiciliaria y } \\
\text { policlínicas }\end{array}$ & $\begin{array}{l}\text { Médico/a de familia o } \\
\text { generalista, enfermero/a, } \\
\text { partero/a, pediatra y } \\
\text { ginecólogo/a visitante } \\
\text { (equipo básico ASSE en } \\
\text { Montevideo) }\end{array}$ & - & $\begin{array}{l}\text { No hay población } \\
\text { adscripta en ASSE }\end{array}$ & - \\
\hline Venezuela & $\begin{array}{l}\text { 6.712 Consultorios Populares } \\
\text { de Barrio Adentro } \\
\text { 4.117 Ambulatorios tipos I y II } \\
608 \text { Ambulatorios Urbanos } \\
\text { III }\end{array}$ & $\begin{array}{l}\text { Médico/a especialista en } \\
\text { medicina general integral, } \\
\text { enfermero/a, Agente } \\
\text { Comunitario/a de Atención } \\
\text { Primaria de Salud (Acap) }\end{array}$ & $\begin{array}{l}\text { Agente Comunitario/a } \\
\text { de Atención Primaria } \\
\text { de Salud (pagado) }\end{array}$ & $\begin{array}{l}\text { Sí, población asignada } \\
\text { por equipo mediante } \\
\text { la dispensarización en } \\
\text { Barrio Adentro }\end{array}$ & $\begin{array}{l}1.250 \text { personas o } \\
250 \text { a } 350 \text { familias } \\
\text { por equipo Barrio } \\
\text { Adentro }\end{array}$ \\
\hline
\end{tabular}

Fuentes: Elaboración propria 


\section{Integración de la APS en la red de servicios}

La integración de la APS en la red de servicios es una preocupación de los policy makers y, cada vez más, los servicios de atención primaria definidos como de primer nivel son establecidos en las normativas oficiales como la puerta preferencial al sistema de salud en los 12 países. Se preconiza que el médico/a/equipo de APS ejerza la función de filtro para acceso a la atención especializada, aunque esta función no siempre esté reglamentada.

La organización de la red con base en la definición de la puerta de entrada preferencial a través de la APS se confronta con la fuerte competencia de los servicios de atención de urgencia y de emergencia hospitalaria y los servicios privados (como en Brasil) como primer contacto y fuente regular de cuidados. En Bolivia, es posible la libre elección de los servicios de salud $y$, en el Uruguay, la función de puerta de entrada puede ser compartida con los hospitales de segundo nivel. Esta es una situación probablemente agravada por la insuficiente articulación entre los puntos de atención (básica y especializada) y por problemas en la organización del proceso de trabajo de los equipos de APS, con baja integración entre el desarrollo de acciones programadas, prácticas promocionales y preventivas y de atención a la demanda espontánea: o sea, la integración entre las acciones para la atención individual y las acciones colectivas de base poblacional son insuficientes.

Los dirigentes también reconocen dificultades para el acceso a la atención especializada. La definición formal de flujos para derivar a los usuarios desde la APS hasta los servicios especializados es una realidad informada por los gestores del sector público de los países, principalmente en los servicios de los nuevos modelos, como en Bolivia, donde hay normas establecidas solamente para el flujo desde el Modelo de Salud Familiar Comunitario Intercultural. La definición formal de flujos no es suficiente para la articulación y la comunicación entre servicios y profesionales de toda la red. La referencia en general funciona con imperfecciones y la contrarreferencia raramente se realiza.

Las dificultades de acceso a los cuidados continuados se expresan en filas y prolongados tiempos de espera para acceso a la atención especializada por la baja oferta pública y la insuficiencia de recursos especializados (consultas y exámenes) en la red en casi todos los países. Aunque hay altos porcentajes de pertinencia de derivación desde la atención primaria a otros niveles de atención, como es el caso de Chile, existen listas de espera para algunas especialidades de oferta más deficitaria. No obstante, los tiempos de espera casi nunca son monitoreados o tornados públicos, con excepción de Uruguay, que define tiempos máximos de espera. Para las especialidades básicas se exige la consulta en 24 horas y para la atención especializada hasta 30 días. Los sistemas de información de Uruguay permiten el monitoreo de los tiempos y su divulgación por parte del Ministerio de Salud.

Se desarrollan iniciativas para mejorar la cualificación y la comunicación entre los profesionales de la APS y otros niveles, como el TeleSalud, en Brasil, y un sistema de citas por teléfono o internet en implementación, en Ecuador.

La integración de la APS en la red depende de acciones a nivel de políticas institucionales que enfrenten la fragmentación de los sistemas de salud con el fortalecimiento de los servicios de APS como puerta de entrada preferencial y de su papel de filtro, además de la ampliación de la oferta de atención especializada, garantizada por el sistema público, con redes territorializadas y ordenadas desde la APS. 


\section{Fuerza de trabajo en APS}

Uno de los principales retos para la prestación y organización de la APS es la disponibilidad de profesionales de salud, sobre todo médicos/as, y la inadecuación de la formación para la APS.

La disponibilidad de profesionales de salud es diversa entre los países, con profundas desigualdades en la oferta y distribución en el interior de cada país y grandes brechas en las zonas remotas y desfavorecidas. La densidad de médicos/as por mil habitantes se distribuye en un amplio rango entre los países. La suficiencia numérica de la oferta parece haber sido alcanzada en Uruguay, con 4,5 médicos por mil habitantes, y Argentina, con 3,8 , así como también en Venezuela, con estimaciones de una razón de 3,8 médicos por mil habitantes en 2014. La insuficiencia de recursos humanos en salud es intensa en Bolivia (0,5 médicos/as/mil hab.), Guyana $(0,9)$, Suriname $(1,0)$ y Perú $(1,1)$. La razón de enfermeros/as profesionales por mil habitantes es menor que la de médicos en siete países (cuadro 6).

No hay estadísticas organizadas de disponibilidad de recursos humanos para la APS específicamente, pero hay consenso entre los gestores nacionales de que la oferta es insuficiente cualitativa y cuantitativamente. En la mayor parte de los países se define que los/ as médicos/as generalistas deben actuar en la APS, pero no se conoce la disponibilidad de médicos/as con formación generalista que actúan en las APS y son escasos los/as médicos/as con especialidad médica para la APS. Esta es más frecuentemente denominada medicina familiar y comunitaria (cuadro 6)

Para enfrentar la insuficiente oferta de médicos en APS y, en algunos casos también de atención especializada, ocho países (Bolivia, Brasil, Ecuador, Guyana, Surinam, Perú, Venezuela y Uruguay) mantienen cooperación con Cuba (cuadro 6).

Uno de los principales problemas para la fijación de los médicos en servicios públicos de APS está en la regulación de las relaciones laborales. Hay diversidad de vínculos laborales y formas de remuneración de la fuerza de trabajo en APS en todos los países, con tendencia a la precarización laboral e inestabilidad con contratos temporarios, sin garantías de beneficios sociales.

Solamente en Chile y Guyana la mayoría de los profesionales de APS son funcionarios públicos. En los otros países se observan combinaciones de contratos temporales con pagos por becas, desempeño o prestación de servicios, contratos por tiempo indeterminado como asalariados del sector privado y funcionarios públicos. Aunque en casi todos los países parte de los profesionales de salud sean funcionarios públicos con cargo presupuestado de duración indefinida, solamente en Chile hay una carrera funcionaria específica en APS.

La inestabilidad de los contratos laborales, con alta rotación de profesionales, dificulta la adhesión e impide la formación de vínculos entre los equipos, las familias y la comunidad. 
Cuadro 6. Fuerza de trabajo en Atención Primaria de Salud en los países suramericanos

\begin{tabular}{|c|c|c|c|c|c|c|}
\hline Países & $\begin{array}{l}\text { Médicos } \\
\text { / } 1000 \\
\text { hab. }\end{array}$ & $\begin{array}{l}\text { Enfermeros } \\
\text { profesionales } \\
\text { / } 1000 \text { hab. }\end{array}$ & $\begin{array}{l}\text { Denominación de la } \\
\text { especialidad médica para } \\
\text { APS } \\
\text { (número de médicos) }\end{array}$ & Vínculos de trabajo en APS & $\begin{array}{l}\text { Carrera } \\
\text { funcionaria } \\
\text { para APS }\end{array}$ & $\begin{array}{l}\text { Presencia de } \\
\text { médicos cubanos } \\
\text { (número) }\end{array}$ \\
\hline Argentina & $\begin{array}{l}3,8 \\
(2010)\end{array}$ & $2,2(2010)$ & $\begin{array}{l}\text { Medicina Familiar y } \\
\text { Comunitaria }\end{array}$ & $\begin{array}{l}\text { Contratos renovables (por evaluación de } \\
\text { desempeño) y temporarios; funcionarios } \\
\text { públicos }\end{array}$ & No & No \\
\hline Bolivia & $\begin{array}{l}0,5 \\
(2014)\end{array}$ & $0,2(2014)$ & $\begin{array}{l}\text { Médico/a especialista en } \\
\text { Salud Familiar Comunitaria } \\
\text { Intercultural } \\
\text { (médico/a general o } \\
\text { familiar) }\end{array}$ & $\begin{array}{l}\text { Funcionarios públicos; } \\
\text { contratos anuales sin estabilidad laboral }\end{array}$ & No & Sí \\
\hline Brasil & $1,9(2010)$ & $1,5(2010)$ & $\begin{array}{l}\text { Medicina de Familia y } \\
\text { Comunidad (3.253) }\end{array}$ & $\begin{array}{l}\text { Funcionarios públicos; asalariados } \\
\text { sector privado; contratos temporarios } \\
\text { sin estabilidad laboral; becas }\end{array}$ & No & Sí (11.300) \\
\hline Chile & $\begin{array}{l}2,0 \\
(2013)\end{array}$ & $1,5(2011)$ & Medicina Familiar (629) & Funcionarios públicos & Sí & $\begin{array}{l}\text { Sí ( } 30 \% \text { de los } \\
\text { médicos APS son } \\
\text { extranjeros, en } \\
\text { parte cubanos) }\end{array}$ \\
\hline Colombia & $1,7(2011)$ & $0,9(2011)$ & $\begin{array}{l}\text { Medicina Familiar (500) } \\
\text { Salud Familiar y } \\
\text { Comunitaria* }\end{array}$ & $\begin{array}{l}\text { Contratos de prestación de servicios a } \\
\text { término fijo (la mayoría) y funcionarios } \\
\text { públicos }\end{array}$ & No & No \\
\hline Ecuador & $1,7(2012)$ & $0,8(2012)$ & $\begin{array}{l}\text { Medicina Familiar y } \\
\text { Comunitaria (112) }\end{array}$ & $\begin{array}{l}\text { Funcionarios públicos; contratos de } \\
\text { corta duración (a partir de 2008) sin } \\
\text { estabilidad laboral }\end{array}$ & No & Sí \\
\hline Guyana & $\begin{array}{l}0,9 \\
(2013)\end{array}$ & $1,5(2013)$ & Medicina General & Funcionarios públicos & No & $\begin{array}{l}\text { Sí ( } 166 \text { médicos/ } \\
\text { as, enfermeros/as } \\
\text { y técnicos/as para } \\
\text { APS y hospitales) }\end{array}$ \\
\hline Paraguay & $1,3(2010)$ & $0,8(2010)$ & Medicina Familiar & $\begin{array}{l}\text { Contratos renovables por evaluación } \\
\text { de desempeño; funcionarios públicos; } \\
\text { salario más bonificación }\end{array}$ & No & No \\
\hline Perú & $1,1(2012)$ & $1,1(2012)$ & Medicina Familiar (119) & $\begin{array}{l}\text { Funcionarios públicos; contrato } \\
\text { periódico de administración de servicios; } \\
\text { asalariados del sector privado }\end{array}$ & No & Sí (APS y AE) \\
\hline Suriname & $1,0(2010)$ & $1,6(2014)$ & $\begin{array}{l}\text { Medicina General } \\
\text { Medicina de Familia (a } \\
\text { partir de 2014) }\end{array}$ & $\begin{array}{l}\text { Contratos de duración indefinida } \\
\text { en el servicio regional (salarios y } \\
\text { complemento con cápita por número } \\
\text { de personas registradas); contratos } \\
\text { por tiempo indeterminado con salario } \\
\text { (Medical Mission). }\end{array}$ & No & Sí (médicos/as) \\
\hline Uruguay & $4,5(2011)$ & $1,4(2010)$ & $\begin{array}{l}\text { Medicina Familiar y } \\
\text { Comunitaria }\end{array}$ & $\begin{array}{l}\text { ASSE: cargos temporales; régimen } \\
\text { derecho privado; funcionarios públicos; } \\
\text { IAMC: asalariados sector privado; } \\
\text { prestación de servicios }\end{array}$ & No & $\begin{array}{l}\text { Sí (oftalmólogos/ } \\
\text { as) }\end{array}$ \\
\hline Venezuela & $\begin{array}{l}3,8 \\
(2010)\end{array}$ & - & $\begin{array}{l}\text { Medicina General Integral } \\
\text { (pregrado en Medicina } \\
\text { Integral Comunitaria: } 20 \\
\text { mil médicos) }\end{array}$ & $\begin{array}{l}\text { Funcionarios públicos; becas (programas } \\
\text { de formación intensiva en Atención } \\
\text { Primaria de Salud) }\end{array}$ & No & Sí \\
\hline
\end{tabular}

Fuentes: Elaboración propria 


\section{Estrategias de formación de la fuerza de trabajo para la APS}

Hay consenso entre los gestores que la formación de profesionales de salud es inadecuada y que no está orientada a la actuación en la APS. En los años recientes se observan en casi todos los países diversas iniciativas de formación para la APS en todos los niveles: técnicos, pregrado, posgrado y desarrollo profesional continuo. Entre las principales iniciativas de formación de la fuerza de trabajo en APS se destacan los posgrados para formar médicos/as especialistas en medicina de familia y comunidad (Argentina, Bolivia, Brasil, Chile, Ecuador, Paraguay, Uruguay y Venezuela) y el programa de formación en Medicina Integral Comunitaria de Venezuela, en cooperación con la Escuela Latinoamericana de Medicina Salvador Allende de Cuba (Ríos, 2014A, 2014B, 2014C; ACOSTA RAMÍREZ; VEGA ROMERO, 2014; ALMEIDA, 2014; VEGA ROMERO; ACOSTA RAMÍREZ, 2014A; TEJERINA SILVA, 2014A, 2014B, 2014C).

Para suplir la carencia de médicos/ as para la APS, desde el 2008 el gobierno venezolano desarrolla un amplio programa nacional de formación en Medicina Integral Comunitaria. En el 2014 estaban matriculados 20 mil estudiantes de medicina integral comunitaria, del primero al sexto año de estudio, con un programa de formación en clínicas académicamente certificadas de Barrio Adentro a cargo de seis universidades venezolanas, con presencia importante de profesores cubanos. Son 6.715 profesores/as médicos/as especialistas en medicina general integral, con componente docente certificado, de la Misión Médica Cubana (VENEZUelA; OPS, 2013; ROJAS, 2014). Hay 16.878 médicos comunitarios graduados y, de estos, 6.285 cursan estudios de posgrado en medicina. Los estudiantes al empezar el curso se comprometen a prestar servicio en el sistema público. Se garantiza a todos los graduados el ingreso al sistema público nacional de salud (ROJAS, 2014). El ejercicio de la medicina por los egresados fue reglamentado por ley en el 2011 (VENEZUELA; OPS, 2013).

En el nivel técnico se destaca la formación de trabajadores de APS alternativos, como en Guyana y Suriname, que tienen tradición en este tipo de trabajadores de salud. Guyana emplea a los/as técnicos/as Medex, que sustituyen a los/as médicos/as en el interior, y Suriname utiliza asistentes de salud también reclutados en los pueblos del interior, que siguen un programa de formación integral de cuatro años para convertirse en trabajadores de la salud de nivel medio (GOEDE, 2014A, 2014B). En el mismo sentido, Ecuador tiene una experiencia más reciente en la formación de Técnico/a en Atención Primaria de Salud (Taps), miembro del Equipo de Atención Integral de Salud (Eais) (TEJERINA SILVA, 2014B).

El reto más importante es la retención de trabajadores de la salud capacitados para ofrecer servicios de salud de calidad a las poblaciones de áreas remotas y/o desfavorecidas. Además de la escasez, la distribución de los trabajadores de la salud es deficiente y favorece las zonas urbanas. Diferentes estrategias son aplicadas por los países para aumentar la disponibilidad de profesionales de la salud en las zonas remotas. Algunos países tienen un período de servicio obligatorio para los recién graduados, con diferentes grados de éxito (como por ejemplo Perú, Paraguay, Guyana, Suriname) y/o incentivos económicos. Diversos países establecieron convenios con Cuba, como lo hizo recientemente Brasil, para suplir la falta de médicos en la APS en zonas desfavorecidas y remotas.

\section{Intersectorialidad}

La interacción con otros sectores (intersectorialidad) para hacer frente a los determinantes sociales y promover la salud es otro componente principal de la APS integral. En diversos países las políticas de salud 
plantean como un eje de la estrategia de APS la promoción de la salud, concebida como acción sobre los determinantes sociales y/o cambios en los estilos de vida, lo que implica políticas con enfoque intersectorial. El paradigma del 'vivir bien' en Bolivia y Venezuela, o el 'buen vivir' en Ecuador, sustenta el accionar de la política pública y puede interpretarse como acción intersectorial por la salud, involucrando un conjunto de políticas de desarrollo social.

En los 12 países de Suramérica pueden identificarse directrices, normas y/o espacios que favorecen la intersectorialidad en APS a nivel local e iniciativas a nivel nacional integrando diversos ministerios, como el Consejo Nacional de Coordinación de Políticas Sociales en Argentina; la Comisión Nacional Intersectorial de Salud Publica en Colombia, con participación de nueve ministerios y tres departamentos nacionales, que tiene el objetivo de orientar intervenciones sobre los determinantes sociales de salud; o el Ministerio Coordinador de Desarrollo Social en Ecuador, encargado de vincular los ministerios de Deporte, Vivienda, Educación, Ambiente, Movilidad Humana y Salud en una nueva división administrativa del país en zonas y distritos para implantar programas intersectoriales.

Los gestores nacionales de APS mencionan programas de transferencias monetarias a grupos vulnerables para reducir la pobreza y combatir el hambre como importantes estrategias intersectoriales. Algunos ejemplos de esto son: en Bolivia, los bonos de salud y educación, la subvención a productos de consumo masivo y el Programa Multisectorial Desnutrición Cero, integrado por 11 ministerios, gobiernos departamentales, municipios y ONG; el bono de desarrollo humano y pensiones no contributivas en Ecuador; el subsidio familiar en Venezuela; el Uruguay Crece Contigo, política nacional con intervenciones focalizadas en zonas de pobreza extrema con metas de salud y nutrición; el Programa Bolsa Familia en
Brasil; o el programa Chile Crece Contigo, que involucra acciones intersectoriales con protagonismo del Ministerio de Salud y del Ministerio de Desarrollo Social en acciones en salud, vivienda y educación.

La intersectorialidad debe abarcar los diversos niveles gubernamentales, desde la esfera nacional hasta los gobiernos municipales y/o provinciales, pero en general no se establece de forma clara cómo se debe realizar la articulación de la acción intersectorial entre los niveles nacionales y subnacionales. Por otra parte, la asignación de los recursos parece ser insuficientes para la efectiva ejecución de las acciones.

En la mayor parte de los países, los equipos de APS deben realizar un diagnóstico del territorio para identificar las condiciones socioeconómicas y de salud y los problemas prioritarios para orientar intervenciones en la comunidad con apoyo de la carpeta familiar y/o el registro de las familias, pero esta acción no siempre es sistemática. Paraguay ha construido una interesante herramienta de integración entre los gobiernos locales y las comunidades llamada Diagnóstico Comunitario Participativo, donde en mesas intersectoriales se hace el análisis de la situación y se designan los problemas de salud prioritarios para las intervenciones (RíOs, 2014B).

Los equipos de APS se relacionan con otros sectores como parte de su actividad extramural. El Agente Comunitario de Salud o los promotores de salud presentes en casi todos los países tienen atribuciones en el diagnóstico comunitario y en la articulación de otras organizaciones y el apoyo a programas de desarrollo social. Cabe mencionar también articulaciones con otros sectores, como educación, desarrollo social, vivienda, medio ambiente, deportes y sanidad.

\section{Participación social}

Las políticas nacionales de salud en Suramérica tienen en general como uno de 
sus pilares la participación de la comunidad, con un cuerpo normativo, políticas y espacios de deliberación, organización y consolidación de la actuación de los ciudadanos. Este escenario fue posible a partir de la transición democrática sucedida en gran parte de los países de la región desde fines del siglo $\mathrm{XX}$, que produjo el inicio de un nuevo ciclo histórico que aportó la perspectiva de la generación de una ciudadanía más autónoma y crítica, así como también articulada con la creación de espacios públicos de participación, conflicto y diálogo (CALDERÓN, 2009). Esta transición construyó las bases para la conformación de espacios institucionalizados de participación de la población en las políticas públicas, como es el caso de la salud.

La participación social se institucionalizó en consejos locales de salud en casi todos los países suramericanos (excepto Suriname y Uruguay, donde está formalizada a nivel nacional), que permiten la participación ciudadana y la articulación entre el Estado, las organizaciones de la sociedad civil y las organizaciones no gubernamentales.

De manera general, la participación social en salud en Suramérica es un espacio formalizado e institucionalizado, incluso en algunos países constitucionalmente, basado en la APS integral y renovada. Pero uno de los obstáculos para la efectividad de la APS integral es que la participación de la comunidad a menudo se limita a la formulación del diagnóstico del territorio e involucra poco a la comunidad en la toma de decisiones para el cambio de políticas que impacten en los determinantes sociales de la salud (LABONTÉ ET AL., 2009).

En los países que comparten una comprensión de la APS renovada, en la cual la participación es uno de los principios de orientación de los sistemas de salud, se consolidó la percepción de que los espacios de deliberación y participación de los ciudadanos se constituyen como instrumentos de democratización de la gestión pública y del aumento de la efectividad de las políticas sociales. No obstante, la institucionalización de la participación presenta límites. No hay una correspondencia entre la conquista de espacios de poder público de participación y el grado de actuación y desarrollo político y organizativo de los sujetos sociales y se observan problemas de representatividad, en la calidad de la participación y en la posibilidad de influir en la deliberación de las políticas (BREILH, 2010; ESCOREL; MOREIRA, 2012).

\section{Interculturalidad}

El enfoque intercultural de los servicios de salud y la integración de la medicina tradicional de los pueblos originarios y campesinos es, cada vez más, parte de las políticas sanitarias nacionales, todavía con implementación muy diferenciada.

Los debates al respecto de la construcción del campo de la 'salud intercultural' y su debido reconocimiento y comprensión de la diversidad étnica en las sociedades es reciente en el campo de la medicina (KNIPPER, 2010). Sin embargo, en Suramérica las experiencias de interculturalidad produjeron en algunos países importantes discusiones e incorporación de aspectos de la medicina de los pueblos originarios en sus sistemas de salud. A pesar de esto, los documentos formales de APS no hacen mención clara al concepto de interculturalidad en la mayoría de los países, con excepción de Bolivia, Chile, Colombia y Perú.

El enfoque intercultural es más desarrollado en Bolivia, donde la concepción de interculturalidad es uno de los pilares de la política nacional de salud y los equipos de atención primaria coordinan acciones con los médicos tradicionales del territorio bajo su responsabilidad. Hay experiencias interculturales localizadas en Chile, Colombia, Ecuador y Perú. Incluso adoptando concepciones y abordajes distintos, estos países utilizan la APS para la implementación de acciones interculturales. 
En la mayor parte de los países de la región la comprensión del enfoque intercultural se restringe a una concepción de respeto a la singularidad e integración sociocultural o establece sus bases dentro de las cuestiones indígenas.

\section{Consideraciones finales}

En síntesis, hoy en América del Sur están en marcha diversos procesos de revitalización de la APS, su implementación es progresiva y en muchos casos aún no se han alcanzado plenamente los resultados esperados. Además de la expansión del acceso a los servicios en el nivel primario, incluso en las experiencias que reducen ese acceso a un conjunto de acciones seleccionadas, es posible identificar movimientos innovadores en la organización y en las prácticas de atención primaria. En algunas de esas experiencias, y en los sistemas públicos mejor consolidados, se observan características de la atención primaria integral.

Es posible destacar inúmeras experiencias innovadoras que pueden propiciar la formulación de políticas para enfrentar problemas similares en la organización y en las prácticas de APS como estrategia para la organización de los sistemas de salud en la región: el enfoque de la salud como un derecho en las constituciones nacionales; las iniciativas de desarrollo del primer nivel, con abordaje individual y de salud colectiva, integrado al sistema de salud con conformación de redes y coordinación con servicios especializados y hospitalarios;las iniciativas de mejoría de la calidad de la atención prestada en el primer nivel; los nuevos abordajes en interculturalidad; la integración horizontal en el territorio con otros servicios públicos y organizaciones de otros sectores no sanitarios que buscan favorecer acciones intersectoriales para enfrentar los determinantes sociales y promover la salud; las experiencias de fuerte acción comunitaria e incidencia en las desigualdades sociales en que la APS está integrada a una política amplia de desarrollo social, como en el paradigma del 'buen vivir' o 'vivir bien', aunque a veces son localizadas o en contextos en los que la segmentación aún no fue superada; la acción comunitaria con la actuación de agentes comunitarios o promotores de salud; la ampliación de la participación social con consejos locales de salud e institucionalización de otras formas de participación en salud; la incorporación a los equipos de APS de personal técnico especializado en APS; y las estrategias de formación de grado y posgrado de profesionales médicos para APS.

Entre los desafíos más importantes para la garantía de los cuidados integrales en salud universales están la formación y el desarrollo profesional continuos para la actuación en APS y para garantizar el acceso a servicios de salud en áreas remotas y desfavorecidas, aunque diversas estrategias para superarlos todavía se encuentren en implementación.

La acción intersectorial ha sido planteada por los movimientos de APS y de promoción de la salud en los últimos 30 años, pero todavía hay muchos obstáculos para la articulación entre los distintos sectores en los países suramericanos. Identificar los problemas que requieren acción intersectorial, articular los distintos intereses de cada sector e indicar los objetivos comunes de las distintas áreas, se configuran aún como importantes desafíos para la organización de la intersectorialidad en la APS en los países analizados en este artículo.

Al reconocer la atención a servicios de salud de calidad como uno de los determinantes sociales de la salud, otro desafío para la garantía de los cuidados integrales en salud está en alcanzar un equilibrio adecuado entre el abordaje individual de cuidados personales en tiempo oportuno y el abordaje de base territorial de salud colectiva, sumado a estrategias de acción comunitaria y cooperación horizontal local. En este sentido, un punto que se necesita profundizar en otras 
investigaciones es cuáles serían las mejores estrategias para lograr implementar de forma articulada un primer nivel fuerte con atención resolutiva de calidad y una acción comunitaria potente para enfrentar los determinantes sociales.

Persisten tensiones entre distintas concepciones de atención primaria entre los países y en el interior de cada país con diversos abordajes en implementación. La segmentación de los sistemas de salud con diferentes coberturas y redes de servicios para grupos poblacionales y la baja financiación pública de los sistemas de salud son importantes obstáculos para una atención primaria integral efectiva. La búsqueda de implementación de una APS verdaderamente integral es indisociable y enfrenta los mismos obstáculos para la construcción de sistemas públicos universales en nuestra región.

En el debate internacional contemporáneo sobre la cobertura universal de salud se observa un embate entre lo que se entiende por derecho a la salud y cómo alcanzar el acceso universal, con correspondencia a distintos abordajes de APS. En este embate se contraponen posiciones polarizadas: cobertura por seguros segmentados versus sistemas públicos universales.

La modalidad de mercado de aseguramiento con coberturas segmentadas por seguros privados o públicos con paquetes de servicios diferenciados conforme la capacidad de pago de las personas, cristalizando desigualdades, se corresponde, por un lado, con un abordaje de APS de primer nivel centrado en la atención individual, sin territorialización ni enfoque colectivo. $\mathrm{Y}$ en el caso de los seguros subsidiados, focalizados (en poblaciones de bajos ingresos o en grupo materno-infantil), con una APS (neo)selectiva con un paquete mínimo de servicios, se termina ofreciendo una atención a la salud pobre para pobres.

Por otra parte, la perspectiva de construcción de sistemas públicos universales de salud para garantía del derecho a la salud y del acceso universal en respuesta a las necesidades individuales y colectivas de salud de los ciudadanos, independientemente de su capacidad de pago, en base a la solidaridad, se corresponde con un abordaje de APS integral como el preconizado en la Declaración de Alma-Ata, una estrategia para reorientar el sistema de salud y garantizar la atención integral. Una APS coordinadora de los cuidados de salud en la red integral, con participación social y actuación intersectorial para enfrentar los determinantes sociales y promover la salud, indisociable del desarrollo económico y social de la nación.

Los abordajes de APS implementados son condicionados por la segmentación de la protección en salud y de los sistemas de salud. En el movimiento de renovación de la APS, las agencias multilaterales propugnan la APS como estrategia para la reorientación de los sistemas de salud, pero lo que se observa es que esto es un proceso de dos vías: los modos como los sistemas de salud son financiados y están organizados orientan los enfoques de Atención Primaria de Salud implementados. La segmentación de coberturas y financiamiento y la fragmentación de la atención, profundizadas en las décadas anteriores, obstaculizan la implementación de la APS integral aun en el contexto de políticas sociales abarcadoras. 


\section{Referências}

ACOSTA RAMÍREZ, N; VEGA ROMERO, R. Mapeo y análisis de los modelos de Atención Primaria en Salud en los países de América del Sur: mapeo de la APS en Perú. Rio de Janeiro: ISAGS: UNASUR, 2014. Disponible en: $<$ http://www.isags-unasur.org/uploads/biblioteca/7/ bb[179]ling[2] anx[552].pdf>. Acceso el: 14 dic. 2014.

ALMEIDA, P. F. Mapeamento e análise dos modelos de Atenção Primária à Saúde nos países da América do Sul: Atenção Primária à Saúde no Brasil. Rio de Janeiro: ISAGS: UNASUR, 2014. Disponible en: <http://www. isags-unasur.org/uploads/biblioteca/7/bb[127]ling[1] anx[519].pdf>. Acceso el: 10 dic. 2014.

BREILH, J. Las tres 'S' de la determinación de la vida: 10 tesis hacia una visión crítica de la determinación social de la vida y la salud. In: NOGUEIRA, R. P. (Org.) Determinação social da saude e reforma sanitaria. Rio de Janeiro: Cebes, 2010. p. 87-125.

CALDERÓN, F. Inflexión histórica: la situación social-institucional en el cambio político de América Latina. In: FLEURY, S.; LOBATO, L. V. C. (Org.). Participação, Democracia e Saúde. Rio de Janeiro: Cebes, 2009. p. 83-100.

CONILL, E. M.; FAUSTO, M. C. R.; GIOVANELLA, L. Contribuições da análise comparada para um marco abrangente na avaliação de sistemas orientados pela atenção primária na América Latina. Rev. Bras. Saúde Matern. Infant., Recife, v. 10, supl. 1, p. s14-s27, 2010.

ESCOREL, S.; MOREIRA, M. R. Participação Social. In: GIOVANELLA, L. et al. (Org.). Políticas e sistema de saúde no Brasil. 2. ed. rev. amp. Rio de Janeiro: Editora Fiocruz: Cebes, 2012. p. 853-883.

GIOVANELLA, L. Los sistemas de salud de Argentina, Brasil y Uruguay en perspectiva comparada. Montevideo: Observatório Mercosur de Sistemas de Salud, 2013.

GIOVANELLA, L. et al. (Org.). Sistemas de salud en Suramérica: desafíos para la universalidad, la integralidad y la equidad. Rio de Janeiro: ISAGS, 2012.

GOEDE, H. Mapeo y análisis de los modelos de Atención Primaria de Salud en los países de América del Sur: mapeo de la APS en Guyana. Rio de Janeiro: ISAGS: UNASUR, 2014a. Disponible en: <http://www. isags-unasur.org/uploads/biblioteca/7/bb[235]ling[2] anx[750].pdf>. Acceso el: 19 dic. 2014.

GOEDE, H. Mapeo y análisis de los modelos de Atención Primaria de Salud en los países de América del Sur: mapeo de la APS en Surinam. Rio de Janeiro: ISAGS: UNASUR, 2014b. Disponible en: <http://www.isags-unasur.org/uploads/biblioteca/7/bb[236]ling[2] anx[751].pdf> Acceso el: 19 dic. 2014.

KNIPPER, M. Más allá de lo indígena: salud e interculturalidad a nivel global. Rev. perú. med. exp. salud publica, Lima, v. 27, n. 1, p. 94-101, 2010.

LABONTÉ, R. et al. Aplicación, efectividad y contexto político de la atención primaria integral de salud: resultados preliminares de una revisión de la literatura mundial. Rev. Gerenc. Polit. Salud, Bogotá, v. 8, n. 16, p. 14-29, 2009.

OLIVER, A. The folly of cross country ranking exercises. Health Economics, Policy and Law, Cambridge, v. 7, n. 1, p. 15-17, 2012.

ORGANIZACIÓN MUNDIAL DE LA SALUD (OMS). Estadísticas Sanitarias Mundiales 2013. Ginebra: OMS, 2012. Disponible en: <http://apps.who.int/iris/bitstream/10665/82218/1/9789243564586_spa.pdf?ua=1>. Acceso el: 29 ene. 2015.

La Atención Primaria en Salud: más necesaria que nunca. Ginebra: OMS, 2008. (Informe sobre la salud en el mundo, 2008).

\section{ORGANIZAÇÃO PAN-AMERICANA DE SAÚDE} (OPS). Renovação da atenção primária nas Américas. Documento de posicionamento da Organização Panamericana de Saúde. Washington, DC: OPS/OMS, 2005.

RÍOS, G. Mapeo y análisis de los modelos de Atención Primaria de Salud en los países de América del Sur: mapeo de la APS en Argentina. Rio de Janeiro: ISAGS: UNASUR, 2014a. Disponible en: <http://www.isags-unasur.org/uploads/biblioteca/7/bb[167]ling[2] anx[548].pdf >. Acceso el: 23 nov. 2014.

Mapeo y análisis de los modelos de Atención Primaria de Salud en los países de América del Sur: mapeo de la APS en Paraguay. Rio de Janeiro: ISAGS: UNASUR, 2014b. Disponible en: <http://www. 
isags-unasur.org/uploads/biblioteca/7/bb[128]ling[2]

anx[530].pdf >. Acceso el: 23 nov. 2014.

Mapeo y análisis de los modelos de Atención Primaria de Salud en los países de América del Sur: mapeo de la APS en Uruguay. Rio de Janeiro: ISAGS: UNASUR, 2014c. Disponible en: <http://www.isags-unasur.org/uploads/biblioteca/7/bb[185]ling[2] anx[559].pdf >. Acceso el: 23 nov. 2014.

ROJAS, D. Medicina Integral Comunitaria: nueve años impulsando la salud preventiva en el pueblo. Caracas, 2014. Disponible en: <http://www.avn.info.ve/contenido/medicina-integral-comunitaria-nueve-a\%C3\%Blos-impulsando-salud-preventiva-pueblo>. Acceso el: 28 ene. 2015.

ROVERE, M. Atención primaria de la salud en debate. Saúde em Debate, Rio de Janeiro, v. 36, n. 94, p. 327-342, 2012

TEJERINA SILVA, H. et al. Reformas de gobiernos socialistas a las políticas de salud en Bolivia y Ecuador: el potencial subestimado de la Atención Primaria Integral de Salud para impactar los determinantes sociales en salud. Medicina Social, Nova York, v. 4, n. 4, p. 273-282, 2009.

TEJERINA SILVA, H. Mapeo y análisis de los modelos de Atención Primaria de Salud en los países de América del Sur: mapeo de la APS en Bolivia. Rio de Janeiro: ISAGS: UNASUR, 2014a. Disponible en: <http://www. isags-unasur.org/uploads/eventos/ev[347]ling[1] anx[325].pdf>. Acceso el: 23 nov. 2014.

Mapeo y análisis de los modelos de Atención

Primaria de Salud en los países de América del Sur: mapeo de la APS en Ecuador. Rio de Janeiro: ISAGS:
UNASUR, 2014b. Disponible en: < http://www.isags-unasursalud.org/uploads/biblioteca/7/bb[186]ling[2] anx[560].pdf>. Acceso el: 23 nov. 2014.

Mapeo y análisis de los modelos de Atención Primaria de Salud en los países de América del Sur: mapeo de la APS en Venezuela. Rio de Janeiro: ISAGS: UNASUR, 2014c. Disponible en: <http://www.isags-unasur.org/uploads/biblioteca/7/bb[178]ling[2] anx[561].pdf >. Acceso el: 23 nov. 2014.

VEGA ROMERO, R; ACOSTA RAMÍREZ, N. Mapeo y análisis de los modelos de Atención Primaria de Salud en los países de América del Sur: mapeo de la APS en Chile. Rio de Janeiro: ISAGS: UNASUR, 2014a. Disponible en: <http://www.isags-unasur.org/uploads/biblioteca/7/ bb[191]ling[2] anx[565].pdf>. Acceso el: 14 dic. 2014.

Mapeo y análisis de los modelos de Atención Primaria de Salud en los países de América del Sur: mapeo de la APS en Colombia. Rio de Janeiro: ISAGS: UNASUR, 2014b. Disponible en: < http://www.isags-unasur.org/uploads/biblioteca/7/bb[160]ling[2] $\operatorname{anx}[521] . p d f>$. Acceso el: 14 dic. 2014.

VENEZUELA. Ministerio del Poder Popular para la Salud; ORGANIZACIÓN PANAMERICANA DE LA SALUD (OPS). Medicina Integral Comunitaria: médicos y médicas para el pueblo. Caracas: MPPS: OPS, 2013.

\footnotetext{
Recebido para publicação em abril de 2014

Versão final em dezembro de 2014

Conflito de interesses: inexistente

Suporte financeiro: não houve
} 